\title{
Análise de estratégias de hedge simultâneo para a produção de soja no Centro-Oeste
}

\author{
Waldemar Antonio da Rocha de Souza ${ }^{1}$ \\ João Gomes Martines-Filho² \\ Pedro Valentim Marques ${ }^{3}$
}

\begin{abstract}
Resumo: Objetivou-se avaliar o hedge simultâneo para a produção de soja do CentroOeste com contratos futuros de preço e taxa de câmbio da BOVESPA/BM\&F, usando um modelo de hedge simultâneo do risco de preços e taxa de câmbio. Calcularam-se as eficiências de diferentes estratégias de hedge. As principais conclusões foram que o hedge simultâneo de risco de preços e taxa de câmbio reduz de forma acentuada o risco da receita total comparativamente ao hedge de preços isolado. A mitigação do risco de taxa de câmbio em conjunto com o de preços é fundamental para uma gestão estratégica dos exportadores de commodities.
\end{abstract}

Palavras-chave: Hedge simultâneo; Risco de preços e taxa de câmbio; Soja.

JEL: G13; Q13; Q14.

1 Doutor em Economia Aplicada pela Universidade de São Paulo (Esalq/USP). Professor Adjunto da Universidade Federal do Amazonas (UFAM). E-mail: warsouza@ufam.edu.br

2 Ph.D em Economia Agrícola pela Ohio State University (EUA). Professor Doutor da Universidade de São Paulo (Esalq/USP). E-mail: martines@usp.br

3 Ph.D em Economia Agrícola pela University of Kentucky (EUA). Professor Titular da Universidade de São Paulo (Esalq/USP). E-mail: pvmarque@esalq.usp.br 


\title{
Analysis of strategies for the simultaneous hedging production of soybeans in the Western Central
}

\begin{abstract}
The aim was to evaluate the simultaneous hedge for the Western Central soybean production with BM\&F-BOVESPA price and exchange rate futures using a model of simultaneous price and exchange rate risk hedge. We calculated the efficiencies of different hedging strategies. The main conclusions were that simultaneously hedging price and exchange rate risk sharply reduces the total revenue risk compared to price hedge alone. The joint risk mitigation of the exchange rate together with price risk is key to strategic management for commodity exporters.
\end{abstract}

Key-words: Simultaneous hedge; Price and exchange rate risk; Soybeans.

JEL: G13; Q13; Q14.

\section{Introdução}

A comercialização de uma safra de commodities agrícolas deve ser avaliada em termos da alocação do portfólio individual do produtor agrícola que objetiva maximizar a sua receita total, minimizando simultaneamente a variância associada. O portfólio ideal do produtor deve consistir de posições da commodity no mercado físico e futuro, com percentuais em cada mercado sinalizados por uma taxa ótima de hedge, ou seja, o percentual da produção negociado no mercado futuro que minimiza a variância da receita total, dadas as condições de mercado.

No caso da soja do Centro-Oeste e de Mato Grosso, em particular, o processo de formação de preços toma por referência principal a praça de Chicago (EUA), onde os valores são explicitados em dólar americano, conforme informação da Associação dos Produtores de Soja e Milho de Mato Grosso (APROSOJA) [comunicação pessoal]. Por seu turno, os contratos futuros de soja da BOVESPA-BM\&F também são expressos em dólar americano.

Assim, o problema decisório enfrentado por um tomador de hedge em MT deve necessariamente incluir a flutuação das taxas cambiais, além do risco de preço, devido à exposição à taxa de câmbio e a elevada participação das exportações na receita total dos produtores.

Dessa forma, uma estratégia eficiente de hedge para os produtores de soja de MT não pode ignorar as variações cambiais, as quais contribuem de forma decisiva para o risco total do portfólio individual do produtor. Logo para uma decisão eficiente de hedge para a produção de soja em MT, deve-se avaliar o hedge simultâneo de preços e taxa de câmbio. 
Neste artigo analisa-se a decisão de se efetuar o hedge simultâneo dos riscos de preço e cambial da produção de soja do Centro-Oeste, utilizando contratos futuros da BOVESPA/BM\&F. A introdução da simultaneidade de hedge de riscos de preço e cambial proporciona melhoria no hedge agregado dos agentes, comparativamente à única posição isolada de hedge do risco de preço, melhorando a renda agregada dos participantes, particularmente os dependentes de receita em moeda estrangeira, como os produtores de soja de MT. O objetivo principal é a análise do grau de risco e a sua redução.

Objetiva-se responder à seguinte questão de pesquisa (research question): em que grau o hedge simultâneo de risco de preços e cambial, usando contratos futuros da $\mathrm{BM} \& \mathrm{~F}$, melhora o resultado final do hedge, em termos de redução da variância da receita total, para produtores de soja do Centro-Oeste, comparativamente ao hedge isolado de risco de preços.

As seguintes perguntas de análise (survey questions) serão exploradas: i) qual é a relação fundamental entre as variáveis econômicas que propiciam a maior eficiência do hedge simultâneo de preços e taxa de câmbio; ii) qual é o grau de eficiência do hedge simultâneo de preços e cambial em termos de redução da variância total do portfólio individual do produtor de soja de MT; e, iii) quais são as estratégias mais eficientes e os resultados probabilísticos de hedge simultâneo de preços e taxa de câmbio, obtidos através de testes empíricos e de simulações de Monte Carlo.

$\mathrm{O}$ artigo divide-se em revisão de literatura, metodologia e dados, resultados e discussão, resumo e conclusões. Faz-se a modelagem do hedge simultâneo de preços e cambial para commodities agrícolas exportáveis e a aplicação do arcabouço em estratégias de minimização de riscos de preços e de câmbio para a soja de MT. Cabe ressaltar que ambos os tópicos, da forma abordada, são inéditos na literatura, onde se insere a contribuição do artigo.

\section{Revisão bibliográfica}

O hedge pode ser caracterizado como o ato de transferência de risco de agentes avessos para especuladores mais tolerantes a risco. A abordagem teórica mainstream considera a motivação econômica com base na lucratividade para modelar o comportamento do tomador de hedge, utilizando-se mercados futuros.

O mercado futuro é um mercado derivativo, existente em conjunto com outros mercados. Segundo Hull (2009, p. 1), um derivativo é um instrumento financeiro cujo valor depende, ou deriva, do valor de outras variáveis básicas subjacentes, em geral os preços de ativos negociados em mercado. 
Working (1953) elaborou a concepção do hedge com fundamentação econômica, através da análise dos objetivos e conseqüências das práticas de operações de hedge no mercado futuro de trigo dos EUA, particularizando a análise para os agentes que mantinham altos estoques e para a operacionalização do hedge, avaliando os seus impactos econômicos. Concluiu que as principais razões para efetur hedge eram: i) facilitar as decisões de compra e venda; ii) dar maior liberdade para as atividades empresariais; iii) proporcionar uma base confiável para armazenagem de excessos de estoques de commodities; e, iv) reduzir os riscos empresariais.

Rolfo (1980) desenvolveu modelo de hedge ótimo para exportadores de cacau sujeitos ao risco de preço e produção, a partir de medidas de expectativas de incertezas sobre os riscos de preço e de produção. Concluiu que o hedge ótimo utilizava poucos contratos futuros, com resultado superior ao hedge simples, onde toda a produção é vendida no mercado futuro.

Chu e Morrison (1984) analisaram a depressão ocorrida nos mercados de commodities primárias em 1981-82, contextualizando historicamente, avaliando as causas dos movimentos e instabilidade dos preços de commodities primárias não-petrolíferas, comparando as tendências de curto e longo prazo e os determinantes dos preços. Concluíram que os preços das commodities primárias não-petrolíferas sofreram mudanças significativas nos padrões de comportamento nos anos 70. As principais variáveis impactantes foram o nível de atividade econômica, com forte influência, a inflação mundial, as taxas cambiais, dólar americano versus demais moedas, os choques de oferta e as taxas de juros.

Thompson e Bond (1985) analisaram os principais fatores que justificavam a participação de traders internacionais de commodities nos mercados futuros americanos. Revisaram a evidência empírica indicativa da magnitude de influência dos fatores de gerenciamento de risco de base e cambial nas decisões de hedge. Tomaram-se por referência as operações de hedge de trigo australiano e de gado canadense nos EUA, concluindo que o hedge cambial tem impacto substancial na decisão de hedge de operadores estrangeiros. As operações de hedge podem ser limitadas a períodos de alta volatilidade de base e a existência de risco de base disseminado.

Em abordagem complementar, Thompson e Bond (1987) examinaram as implicações da variabilidade da taxa de câmbio sobre o hedge ótimo de commodities, através da derivação da taxa ótima de hedge nos EUA, num arcabouço teórico de média-variância, pela análise das decisões de hedge de traders estrangeiros para estabilizar a receitas em moeda nacional, sujeitas à variação cambial, através da metodologia VAR, comparando traders australianos e americanos em termos do número de contratos de risco de preço, com e sem hedge cambial. 
Concluíram que o risco cambial pode afetar as decisões de hedge das commodities quando os preços e câmbio são relacionados no tempo, existindo potencial de hedge de risco de preço e cambial nos mercados futuros e a termo. Sem o hedge cambial, o número de contratos futuros do tomador de hedge para trigo australiano seria o dobro do tomador americano, com o hedge cambial o número de contratos é aproximadamente o mesmo.

Novak e Unterschultz (1996) apresentaram metodologia simplificada para mensurar a redução de risco de preço total de curto prazo usando contratos futuros de commodities no exterior. O risco de preço de curto prazo foi decomposto em riscos de preço futuro, de base e cambial, através de previsões "perfeitas" usando testes empíricos de medida de risco com base no erro quadrático médio (EQM), aplicado a um pecuarista canadense que opere contratos futuros nos EUA para hedge. Concluíram que, para o pecuarista canadense, o risco cambial era baixo, não sendo afetado significativamente pelo hedge da taxa de câmbio, e que o hedge de preço neutraliza aproximadamente $60 \%$ do risco e $39 \%$ do risco de base.

Fernandez (2006) avaliou os benefícios potenciais de estratégias de minimização de risco cambial e de inflação nos mercados futuros chilenos, através do hedge simultâneo de risco de preços e cambial. Simulando diferentes cenários de risco cambial, obteve que a maior parte do risco cambial poderia ser reduzida através de estratégias de rolagem contínua com contratos futuros de câmbio de peso chileno e dólar americano.

A literatura acadêmica sobre a utilização dos mercados futuros de soja baseia-se na avaliação da efetividade do hedge de risco de preço. Nesse sentido, Aguiar e Martins (2004) avaliaram a efetividade do hedge dos contratos futuros da CBOT em diferentes vencimentos para os operadores brasileiros de soja em grão de diferentes regiões, através do cálculo do quadrado da correlação entre os preços a vista e futuro.

Concluíram que as cotações dos contratos futuros, com vencimento no segundo semestre, seriam mais influenciadas pela safra norte-americana e as do primeiro semestre, pelo volume de estoque do grão. Além disso, os hedgers localizados mais próximos aos portos de embarque de soja obteriam maior efetividade do hedge. Observaram que seria recomendável aos hedgers brasileiros utilizarem contratos futuros, de julho ou agosto, em suas operações.

Maia e Aguiar (2010) analisaram, a partir do enfoque da base, os retornos e os riscos de estratégias de hedge com contratos futuros de soja em grão da CBOT, nas principais regiões produtoras do país. Formularam quadros identificadores dos retornos médios e riscos esperados, dependendo do período operacional do hedge, do vencimento do contrato futuro escolhido e da duração da operação. 
Concluíram que apesar de variações numéricas entre regiões e vencimentos dos contratos futuros, a base entre os preços à vista no Brasil e as cotações a futuro da CBOT apresentam um padrão definido, com fortalecimento entre maio e novembro, seguido por enfraquecimento nos seis meses subsequentes. Tal fato sugeriu que, em média, operações de hedge de venda só devessem ser assumidas entre os meses de maio e novembro. As operações de hedge de compra só deveriam ocorrer nos demais meses.

A principal referência metodológica deste artigo é Nayak e Turvey (2000), os quais desenvolveram modelagem teórica para a obtenção da taxa ótima de hedge simultâneo de riscos de preço, produtividade e cambial, com dados de produtores de milho canadenses operando contratos futuros nos EUA. Simulou-se a efetividade do hedge simultâneo, avaliando com um modelo teórico quantitativo e simulações de Monte Carlo, em vários cenários alternativos. Concluíram que um produtor estrangeiro que faça hedge simultâneo de preço, produtividade e cambial obterá significativas reduções de risco, do que simplesmente fazendo hedge de risco de preço.

A contribuição diferenciada do presente artigo é a modelagem do hedge simultâneo de preços e cambial para commodities agrícolas, analisando a sua efetividade empiricamente no mercado de soja de Mato Grosso e através de simulações de Monte Carlo, destacando-se o ineditismo da abordagem.

\section{Referencial metodológico e dados}

O produtor de soja do Centro-Oeste que opere na BOVESPA/BM\&F como tomador de hedge de preços no mercado futuro de soja deve adicionalmente projetar a expectativa de variação cambial futura, para avaliar o hedge em contratos futuros de taxa de câmbio. Na BOVESPA/BM\&F são negociados contratos futuros de dólar americano, o qual é a principal moeda referência de valor para o mercado de soja de MT.

\subsection{O modelo}

Com base em Nayak e Turvey (2000), desenvolve-se modelagem teórica de hedge simultâneo de preços e cambial para commodities agrícolas cujos mercados sejam afetados pelo risco de câmbio, além do risco de preços. O modelo será testado empiricamente com dados de preços nos mercados a vista em MT e futuros de preços de soja e dólar americano na BOVESPA/ $\mathrm{BM} \& \mathrm{~F}$, além de simulações probabilísticas das trajetórias das estratégias de hedge através do método de Monte Carlo. 
Por hipótese o produtor agrícola individual detém um conjunto fixo de oportunidades de produção, tal que a decisão de plantio é feita exogenamente e não é afetada pelos preços futuros, isolando-se o problema de decisão de hedge de preços e cambial. Considera-se apenas o processo de decisão de um período, com a decisão de hedge sendo feita no início do período e a posição em contratos futuros fechada no final. O mercado é líquido e transparente e os contratos são divisíveis em qualquer tamanho.

De maneira análoga a Nayak e Turvey (2000), desconsideraram-se os custos de transação no estudo. Entretanto, segundo Campbell et al. (1997, p. 314), a existência de diversos custos operacionais, tais como spread de compra e venda, custo de execução de ordens, taxas e outros, caracterizam-se como fricções de mercado, limitando a capacidade de exploração dos padrões financeiros identificados empiricamente. Tal fato implica na interpretação dos resultados analíticos com certa cautela.

A seguir explicitam-se as variáveis do modelo, com minúsculas indicando as variáveis aleatórias, as maiúsculas as variáveis determinísticas, adotando-se a seguinte notação:

$A$ = volume de produção plantado de determinado produto;

$p=$ preço a vista local no final do período;

$h=$ posição no mercado futuro da commodity;

$F_{1}=$ preço futuro da commodity no início do período;

$f_{2}=$ preço futuro commodity no final do período;

$c=$ posição no mercado futuro de taxa de câmbio;

$E_{1}=$ taxa de câmbio no início da posição no mercado futuro de câmbio;

$e_{2}=$ taxa de câmbio no final da posição no mercado futuro de câmbio;

$e_{\mathrm{r}}=$ taxa de câmbio no mercado a vista no final do período.

O fluxo de receita total do produtor agrícola no final do período, baseado na decisão de fazer hedge do risco de renda operando contratos futuros de preço e taxa de câmbio, expresso em moeda local, será dado por :

$$
H R=R+h\left(F_{1}-f_{2}\right) e_{r}+c\left(E_{1}-e_{2}\right)
$$

Onde:

$H R=$ receita incluindo posição de hedge;

$R=A \times p=$ receita a vista no final do período. 
Ambas as variáveis $R$ e $H R$ têm natureza estocástica no início do período quando a decisão de hedge é feita. Definindo-se:

$$
\begin{aligned}
& f=F_{1}-f_{2} \\
& e=E_{1}-e_{2}
\end{aligned}
$$

A equação (1) pode ser reescrita como:

$$
H R=R+h f e_{\mathrm{r}}+c e
$$

A variância da receita com posição de hedge pode ser escrita como:

$$
s_{H R}^{2}=s_{R}^{2}+h^{2} s_{f e r}^{2}+c^{2} s^{2}+2 h s_{R, f e r}+2 c s_{R, e}+2 h c s_{f e r, e}
$$

Onde:

$$
\begin{aligned}
& S_{R}^{2}=\operatorname{Var}(R) ; \\
& S_{f e r}^{2}=\operatorname{Var}\left(f e_{r}\right) ; \\
& S^{2}=\operatorname{Var}(e) ; \\
& s_{R_{f} f e r}=\operatorname{Cov}\left(R, f e_{r}\right) ; \\
& s_{R, e}=\operatorname{Cov}(R, e) ; \\
& s_{f e r, e}=\operatorname{Cov}\left(f e_{r}, e\right) .
\end{aligned}
$$

As variâncias e covariâncias dos termos compostos são calculadas a partir das médias, variâncias e covariâncias das variáveis originais.

A decisão de hedge é feita através da minimização da variância da receita com posição de hedge na equação (3) com relação a $h$ e $c$, as posições nos mercados futuros de preços e taxa de câmbio, respectivamente. As posições de venda ou compra nos mercados futuros derivam dos sinais de $h$ e $c$. Se $h$ é positivo, o tomador de hedge está vendido em contratos de preços. Se $c$ é negativo, a posição é comprada em contratos de taxa de câmbio.

As condições de primeira ordem são dadas por:

$$
\begin{gathered}
\frac{\partial \sigma_{\mathbb{H}}^{2}}{\partial h}=2 h s_{f e r}^{2}+2 c s_{f e r, e}+2 s_{R, f e r}=0 \\
\frac{\partial \sigma_{\mathbb{H}}^{2}}{\partial c}=2 c s^{2}{ }_{e}+2 h s_{f e r, e}+2 s_{R, e}=0
\end{gathered}
$$

Rearranjando as equações (4.1) e (4.2), tem-se que :

$$
\begin{gathered}
h s_{f e r}^{2}+c s_{f e r, e}=-s_{R, f e r} \\
h s_{f e r, e}+c s^{2}=-s_{R, e}
\end{gathered}
$$


A estratégia de hedge da firma é determinada resolvendo-se o sistema de equações acima para $h$ e $c$. As posições de hedge de risco mínimo em contratos futuros de preços e taxa de câmbio são dadas por:

$$
\begin{gathered}
h_{p e}^{R M}=\frac{1}{1-\rho_{f e r, e}^{2}}\left(-\frac{\sigma_{R, f e r}}{\rho_{f e r}^{2}}+\frac{\sigma_{R, e} \sigma_{f e r, e}}{\sigma_{f e r}^{2} \sigma_{e}^{2}}\right) \\
c_{p e}^{R M}=\frac{1}{1-\rho_{f e r, e}^{2}}\left(-\frac{\sigma_{R, e}}{\rho_{e}^{2}}+\frac{\sigma_{R, f e r} \sigma_{f e r, e}}{\sigma_{f e r}^{2} \sigma_{e}^{2}}\right)
\end{gathered}
$$

Onde: $\rho_{\text {fer }, e}^{2}=\frac{\rho_{\text {fer }, e}^{2}}{\rho_{\text {fer }}^{2} \rho_{e}^{2}}=$

o quadrado do coeficiente de correlação entre os preços futuros, expressos em moeda local. e precos dos contratos futuros de taxa de câmbio.

O primeiro termo entre parênteses do hedge de preços, na equação (6.1), indica a posição dos contratos futuros necessária para minimizar a variabilidade da receita associada às flutuações em moeda nacional. O segundo termo decorre da presença de risco cambial no uso de contratos futuros, considerando a covariância entre os preços locais do mercado a vista e a taxa de câmbio, e também dos preços futuros e da taxa de câmbio futura.

A decisão de minimizar hedge de um produtor agrícola na presença de risco cambial sinaliza que as variâncias e covariâncias avaliadas entre a receita no mercado a vista e os preços futuros em moeda nacional não são iguais às variâncias e covariâncias expressas em moeda estrangeira.

Para exemplificar, tem-se $s_{R, f e r}$, a covariância entre a receita e os preços futuros em moeda nacional, onde a $R$ é a receita a vista no final do período, $f$ indica o diferencial entre a taxa de câmbio atual e futura, expresso em moeda nacional, e $e_{r}$ define a taxa de câmbio no mercado a vista no final do período.

\subsection{Valores do hedge e da redução de risco}

Os hedges de risco mínimo são derivados a partir das avaliações das covariâncias entre preço e taxa de câmbio a vista, bem como do preço e taxa de câmbio futuros. Diversas combinações de instrumentos de hedge são avaliadas para administração do risco da receita (variância) usando mercados futuros, conforme explicitado no Quadro 1: 
QUADRO 1. NÍVEIS DE HEDGE POSSÍVEIS PELA COMBINAÇÃO DE DERIVATIVOS

\begin{tabular}{|l|l|}
\hline \multicolumn{1}{|c|}{ Objetivos } & \multicolumn{1}{|c|}{ Ajustes nas Equações } \\
\hline Hedge de preço e cambial & Eqs. 6.1 e 6.2 inalteradas \\
\hline Hedge de preço & $s_{R, e}=$ o e $s_{\text {fer,e }}=$ o na eq. (6.1) \\
\hline Hedge cambial & $s_{R, f e r}=$ o e $s_{\text {fer }, e}=$ o na eq. (6.2) \\
\hline
\end{tabular}

FONTE: Adaptado de Nayak e Turvey (2000).

Percebe-se que uma desvalorização cambial da moeda nacional eleva os preços das commodities expressos em moeda nacional, o que implica que as relações de covariância dever ser consideradas nas decisões de hedge. Porém não se avaliam os efeitos da covariância entre os preços e a taxa de câmbio sobre a magnitude da taxa de hedge.

\subsection{O uso dos contratos futuros de preços e cambial}

A avaliação do hedge é feita de forma indireta pela obtenção de medidas de sua efetividade (hedge effectiveness). No caso do hedge de variância mínima, a efetividade é medida pela redução na variância da receita. Os hedges de variância mínima de preços e taxa de câmbio foram obtidos pelas equações (6.1) e (6.2).

A redução absoluta de risco (variância) é dada pela diferença entre a variância da receita sem hedge e com hedge ao se utilizarem ambos os contratos futuros:

$$
\begin{aligned}
& \sigma_{R}^{2}-\left(\sigma_{H R p e}^{R M}\right)^{2}= \\
& {\left[\left(h_{p e}^{R M}\right)^{2} \sigma_{f e r}^{2}+\left(c_{p e}^{R M}\right)^{2} \sigma_{e}^{2}+2 h_{p e}^{R M} \sigma_{R, f e r}+2 c_{p e}^{R M} \sigma_{R, e}+2 h_{p e}^{R M} c_{p e}^{R M} \sigma_{f e r, e}\right]}
\end{aligned}
$$

Onde as variáveis foram definidas anteriormente.

Com o uso apenas de contratos de preço, a redução de risco (variância) será dada por:

$$
\sigma_{R}^{2}-\left(\sigma_{H R_{p}}^{R M}\right)^{2}=\left[h_{1}^{2} \sigma_{f e r}^{2}+2 h_{1} \sigma_{R, f e r}\right]
$$

Onde:

$$
h_{1}=-\frac{\sigma_{R, f e r}}{\sigma_{f e r}^{2}}
$$

A equação (9) representa a redução da variância dada pelo hedge isolado de preços, $\sigma_{R}^{2}=\left(\sigma_{H R p}^{R M}\right)^{2}$. O valor de $h_{1}$ indica o percentual da produção total que deve ser negociado no mercado futuro de soja, com contratos da BOVESPA/ $\mathrm{BM} \& \mathrm{~F}$. 
Com o uso apenas de contratos de taxas de câmbio, a redução de risco (variância) será dada por:

$$
\sigma_{R}^{2}-\left(\sigma_{H R_{e}}^{R M}\right)^{2}=\left[c_{1}^{2} \sigma_{e}^{2}+2 c_{1} \sigma_{R, e}\right]
$$

A equação (10) representa a redução da variância dada pelo hedge isolado de câmbio, $\sigma_{R}^{2}-\left(\sigma_{H R_{e}}^{R M}\right)^{2}$. O valor de $c_{1}$ indica o número de contratos que deve ser negociado no mercado futuro de câmbio na BOVESPA/BM\&F.

A magnitude da redução de risco depende da correlação e covariância entre os resultados aleatórios. O modelo teórico será usado para calcular os níveis de hedge de risco mínimo e de redução de risco.

Dentre as hipóteses do modelo teórico de hedge simultâneo encontrase a hipótese básica de não-viés dos preços futuros, ou seja, $F_{1}=E\left(f_{2}\right)$ e $E_{1}=E\left(e_{2}\right)$, para os preços e taxa de câmbio, respectivamente. $O$ ganho esperado de se operar nos mercados futuros é nulo, por hipótese. Como resultado, a receita esperada é independente dos níveis de hedge e o objetivo do agente é minimizar o risco, mensurado pela variância.

Serão avaliadas as posições de hedge de preços isolado, cambial isolado e de preços e cambial simultâneo, através da análise da redução de risco (variância), com o uso de contratos futuros. Os preços são considerados estocásticos e o hedge da receita resultante é feito usando-se a combinação acima, através da matriz de variância-covariância entre a receita total, o hedge de preços, o hedge cambial e o hedge simultâneo de preços e cambial.

\subsection{Estratégias de hedge e resultados probabilísticos}

Para se obter inferências adicionais sobre as diversas estratégias de hedge, serão feitas simulações de Monte Carlo com as taxas de hedge explicitadas, usando-se a matriz de variância-covariância das taxas de hedge. Por hipótese a matriz de covariâncias é normal multivariada conjunta (joint multivariate normal) e os custos de transação são nulos. Os detalhes e deduções matemáticas estão disponíveis por solicitação aos autores.

\subsection{Dados utilizados}

Foram utilizados quatro conjuntos de dados:

1) preços a vista em Rondonópolis (MT), fonte Esalq/CEPEA, dados diários, em $\mathrm{R} \$ /$ saca de 60 kgs.;

2) preços dos contratos futuros de soja da BOVESPA/BM\&F, dados diários, em US\$/saca de 60 kgs.; 
3) preços dos contratos futuros de dólar americano da BOVESPA/BM\&F, dados diários, em R\$/US\$;

4) taxa de câmbio a vista, cotação de venda PTAX-80o, do Banco Central do Brasil, em R\$/US\$.

Carchano e Pardo (2009) mostraram que dentre cinco diferentes metodologias usadas para construir séries contínuas de índices de contratos futuros, com objetivos acadêmicos e operacionais, não existem diferenças significativas entre as séries resultantes, concluindo que pode ser utilizado o método com o menor grau de complexidade.

Dessa forma, para obtenção da série de preços contínua para os contratos futuros de soja da BOVESPA/BM\&F, são considerados o mês de vencimento e o último dia de negociação do contrato para a construção de intervalos de tempo sucessivos, sem interseção entre si. A data de rolagem, i. e., o ponto no tempo em que a série de um contrato é trocada pela de outro, é o dia útil anterior ao vencimento do contrato.

Para todas as séries de dados acima, calculam-se as médias mensais entre Março de 2004 e Maio de 2009. Os dados dos preços a vista em Rondonópolis (MT), foram usados em R\$ e US\$/saca de 6okgs, conversão pela taxa de câmbio a vista, cotação de venda PTAX-80o, do Banco Central do Brasil, em R\$/US\$.

Conforme Nayak e Turvey (2000), usa-se a hipótese fundamental de não viés nos preços futuros, ou seja, $F_{1}=E\left(f_{2}\right)$, e no câmbio futuro, $E_{1}=E\left(e_{2}\right)$. O lucro esperado por operar em ambos os mercados futuros é, por hipótese, nulo. A receita esperada independe dos níveis de hedge e o objetivo do agente é minimizar o risco, medido pela variância.

\section{Resultados e discussão}

Apresentam-se os principais resultados utilizando-se o arcabouço metodológico descrito na seção anterior.

\subsection{Estatísticas dos dados utilizados nas análises empíricas}

No Gráfico 1 explicitam-se, para fins comparativos, as médias mensais dos preços da soja a vista em Rondonópolis (MT), em reais e dólares americanos, bem como os preços futuros da BOVESPA/BM\&F, em dólares americanos, por saca de $60 \mathrm{kgs}$ : 
GRÁFICO 1. SOJA: PREÇOS A VISTA EM RONDONÓPOLIS (MT) E PREÇOS FUTUROS (BOVESPA/BM\&F). MÉDIAS MENSAIS - MAR./04 A MAIO/o9

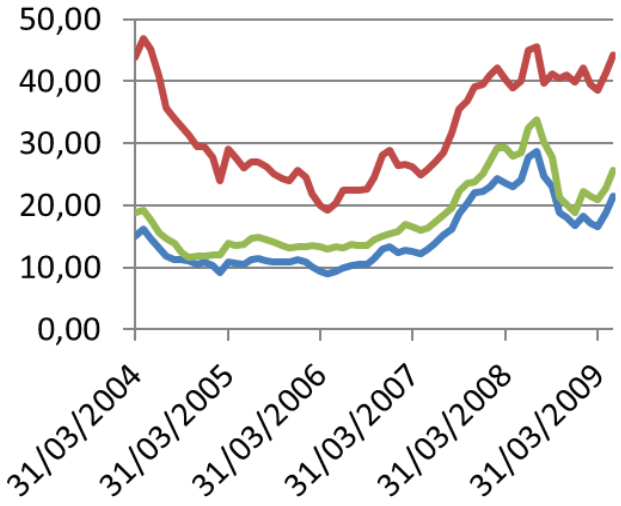

Rondo cash US\$

Rondo cash R\$

BMF futs US\$

FONTE: dados da pesquisa.

Na Tabela 1 apresenta-se a matriz de variância e covariância entre as séries de preços, utilizadas na obtenção das razões ótimas de hedge simultâneo, conforme as variáveis descritas na seção de Metodologia.

TABELA 1. MATRIZ DE VARIÂNCIA E COVARIÂNCIA DOS DADOS MENSAIS. MÉDIAS MENSAIS - MAR./O4 A MAIO/o9

\begin{tabular}{l|c|c|c|c|c}
\hline & $\begin{array}{c}\text { US\$ } \\
\text { FUTS }\end{array}$ & $\begin{array}{c}\text { US\$ } \\
\text { SPOT }\end{array}$ & $\begin{array}{c}\text { Rondo cash } \\
\text { US\$ }\end{array}$ & $\begin{array}{c}\text { Rondo cash } \\
\text { R\$ }\end{array}$ & $\begin{array}{c}\text { BMF futs } \\
\text { US } \$\end{array}$ \\
\hline US\$ FUTS & 0.16 & 0.16 & -1.31 & -0.25 & -1.56 \\
US\$ SPOT & 0.16 & 0.16 & -1.29 & -0.25 & -1.54 \\
Rondo cash US\$ & -1.31 & -1.29 & 27.78 & 34.01 & 30.41 \\
Rondo cash R\$ & -0.25 & -0.25 & 34.01 & 64.58 & 36.44 \\
BMF futs US\$ & -1.56 & -1.54 & 30.41 & 36.44 & 35.19 \\
\hline
\end{tabular}

FONTE: dados da pesquisa. Obs.: US\$ FUTS: taxa do dólar americano futuro na BOVESPA/BM\&F, em R\$/ US\$; US\$ SPOT: taxa de venda do dólar americano a vista, PTAX-8oo, do BACEN; Rondo cash US\$: preço a vista da saca de $60 \mathrm{kgs}$ de soja em Rondonópolis (MT), em US\$/sc; Rondo cash R\$: preço a vista da saca de $60 \mathrm{kgs}$ de soja em Rondonópolis (MT), em R\$/sc; BMF futs US\$: preço do contrato futuro de soja na BOVESPA-BM\&F, em US\$/sc de $60 \mathrm{kgs}$. 
TABELA 2. COEFICIENTES DE CORRELAÇÃO DOS DADOS DAS MÉDIAS MENSAIS - MAR./O4 A MAIO/o9

\begin{tabular}{l|c|c|c|c|c}
\hline & US\$ FUTS & US\$ SPOT & $\begin{array}{c}\text { Rondo cash } \\
\text { US\$ }\end{array}$ & $\begin{array}{c}\text { Rondo cash } \\
\text { R\$ }\end{array}$ & $\begin{array}{c}\text { BMF futs } \\
\text { US\$ }\end{array}$ \\
\hline US\$ FUTS & 1.00 & 1.00 & -0.62 & -0.08 & -0.66 \\
US\$ SPOT & 1.00 & 1.00 & -0.62 & -0.08 & -0.66 \\
Rondo cash US\$ & -0.62 & -0.62 & 1.00 & 0.82 & 0.99 \\
Rondo cash R\$ & -0.08 & -0.08 & 0.82 & 1.00 & 0.78 \\
\hline
\end{tabular}

FONTE: dados da pesquisa. Obs.: US\$ FUTS: taxa do dólar americano futuro na BOVESPA/BM\&F, em R\$/ US\$; US\$ SPOT: taxa de venda do dólar americano a vista, PTAX-80o, do BACEN; Rondo cash US\$: preço a vista da saca de 60 kgs de soja em Rondonópolis (MT), em US\$/sc; Rondo cash R\$: preço a vista da saca de $60 \mathrm{kgs}$ de soja em Rondonópolis (MT), em R\$/sc; BMF futs US\$: preço do contrato futuro de soja na BOVESPA-BM\&F, em US\$/sc de 60 kgs.

Observa-se a baixa e negativa correlação (-0.08) entre a taxa de câmbio futuro e os preços a vista em R $\$$ em Rondonópolis (MT). Também destacam-se as altas e negativas correlações $(-0,6)$ entre as taxas de câmbio a vista e futura e os contratos futuros de soja da BOVESPA/BM\&F. Tais ocorrências podem ajudar a explicar o grau de efetividade do hedge simultâneo.

TABELA 3. ESTATÍSTICAS DESCRITIVAS DOS DADOS MENSAIS. MÉDIAS MENSAIS - MAR./O4 A MAIO/o9

\begin{tabular}{l|c|c|c|c|c}
\hline & US\$ FUTS & US\$ SPOT & $\begin{array}{c}\text { Rondo cash } \\
\text { US } \$\end{array}$ & $\begin{array}{c}\text { Rondo cash } \\
\text { R } \$\end{array}$ & $\begin{array}{c}\text { BMF futs } \\
\text { US } \$\end{array}$ \\
\hline MÉDIA & 25 & 24 & 14.95 & 323 & 18.38 \\
DESV PAD & 0.40 & 0.40 & 5.27 & 8.04 & 5.93 \\
COEF VAR & 0.18 & 0.18 & 0.35 & 0.25 & 0.33 \\
\hline
\end{tabular}

FONTE: dados da pesquisa. Obs.: US\$ FUTS: taxa do dólar americano futuro na BOVESPA-BM\&F, em R\$/US\$; US\$ SPOT: taxa de venda do dólar americano a vista, PTAX-8oo, do BACEN; Rondo cash US\$: preço a vista da saca de $60 \mathrm{kgs}$ de soja em Rondonópolis (MT), em US\$/sc; Rondo cash R\$: preço a vista da saca de 60 kgs de soja em Rondonópolis (MT), em R\$/sc; BMF futs US\$: preço do contrato futuro de soja na BOVESPA-BM\&F, em US\$/sc de $60 \mathrm{kgs}$.

Observa-se que o alto coeficiente de variação do preço a vista da soja em MT em US\$ (0.35) pode indicar forte variância da receita total em dólares americanos, sinalizando a necessidade de uso de mecanismos de mitigação de risco dos preços cotados em moeda estrangeira. 


\subsection{Matriz de variância e covariância utilizada no cálculo dos níveis de hedge e redução de risco}

Tem-se que, seguindo Nayak e Turvey (2002):

$$
f e_{r}=\left(F_{1}-f_{2}\right) e_{r}
$$

Onde:

$F_{1}=$ preço do contrato futuro de soja na BM\&F/BOVESPA, média do mês $t$, em US\$,

$f_{2}=$ média dos preços futuros do período Abr./04 a Maio/2009 (US\$ 18.38), $e_{r}=$ média das taxas de câmbio a vista do período Abr./04 a Maio/o9 (R\$24).

$$
e=E_{1}-e_{2}
$$

Onde:

$E_{1}=$ taxa de câmbio futuro na BM\&F/BOVESPA, média do mês $t$, em R $\$$,

$e_{2}=$ média das taxas de câmbio a vista do período Abr./04 a Maio/o9 (R \$ 24).

A partir das relações indicadas obtém-se a matriz de variância e covariância e os coeficientes de correlação entre as variáveis explicitadas, usados no cálculo das razões de hedge simultâneo ótimas conforme as Tabelas 4 e 5:

TABELA 4. MATRIZ DE VARIÂNCIA E COVARIÂNCIA

\begin{tabular}{l|c|c|c}
\hline & Receita Total & $f e_{r}$ & $e$ \\
\hline Receita Total & 641 & 81.90 & -0.53 \\
$f e_{r}$ & 81.90 & 174.75 & -49 \\
$e$ & -0.53 & -49 & 0.17 \\
\hline
\end{tabular}

FONTE: dados da pesquisa.

TABELA 5. COEFICIENTES DE CORRELAÇÃO

\begin{tabular}{l|c|c}
\hline & $f e_{r}$ & $e$ \\
\hline Receita Total & 0.79 & -0.16 \\
$f e_{r}$ & 1.00 & -0.66 \\
\hline
\end{tabular}

FONTE: dados da pesquisa. 


\subsection{Níveis de hedge de variância mínima e reduções de risco (variância)}

Avaliam-se diversas combinações de instrumentos de hedge disponíveis na BOVESPA/BM\&F, para reduzir o risco da receita total (variância) do produtor de soja de MT. Indicam-se os níveis de hedge em $\mathrm{R} \$ /$ saca de 60 kgs. Na Tabela 6 comparam-se as diversas estratégias de hedge e a reduções da variância da receita total do produtor de soja de MT, obtidas através das Equações (8), (9) e (10):

TABELA 6. REDUÇÃO DA VARIÂNCIA DA RECEITA TOTAL EM VALOR ABSOLUTO (R \$/SACA DE 6o KGS) E PERCENTUAL

\begin{tabular}{l|c|c}
\hline \multicolumn{1}{c|}{ Estratégia Hedge } & Valor Absoluto & $\%$ \\
\hline Preços apenas & 38.38 & $60.5 \%$ \\
Câmbio apenas & 1.67 & $6 \%$ \\
Preços e Câmbio & 51.16 & $80.7 \%$ \\
\hline
\end{tabular}

FONTE: dados da pesquisa.

A eficiência do hedge é medida pela redução da variância da receita, considerando-se estocásticos os preços e taxas de câmbio a vista e futuros. Comparando-se diferentes mix de instrumentos de hedge conforme a Tab. 6, observa-se que a estratégia de hedge com maior impacto sobre a variância é a de hedge simultâneo de risco de preços e de câmbio, com o uso simultâneo de contratos de câmbio e futuros de soja da BOVESPA/BM\&F. O resultado está em linha com o encontrado por Nayak e Turvey (2000).

\subsection{Estratégias de Hedge e Simulações Probabilísticas de Monte Carlo}

Segundo Boyle et al. (1997), as simulações de Monte Carlo são úteis em várias modelagens financeiras, tais como a valoração e estimação de sensibilidade de títulos, análise de risco e testes de stress de portfólios.

Campbell et al. (1997, p.385) observaram que estimadores discretos gerados por simulações de Monte Carlo aproximavam-se assintoticamente de estimadores contínuos, necessitando-se apenas calibrar o número de observações e repetições.

Conforme Nayak e Turvey (2000) fizeram-se simulações de Monte Carlo para obter maiores inferências sobre as diversas estratégias estocásticas de hedge, gerando-se 1.000 (mil) iterações e considerando a matriz de variânciacovariância entre $R, f e_{r}$ e $e$ como sendo normal multivariada conjunta. Os resultados da simulação estão na Tabela 7: 
TABELA 7. SIMULAÇÃO DE MONTE CARLO DAS ESTRATÉGIAS DE HEDGE

\begin{tabular}{l|c|c|c|c}
\hline & Sem Hedge & Preços & Câmbio & Simultâneo \\
\hline Mínimo & 10.88 & 14.30 & 8.95 & 21.04 \\
Máximo & 59.30 & 49.23 & 60.50 & 48.40 \\
Média & 35.30 & 33.30 & 31.67 & 32.90 \\
Desvio Padrão & 7.74 & 4.88 & 7.90 & 2.00 \\
Coef. Variação & 0.24 & 0.15 & 0.25 & 0.09 \\
Variância & 59.84 & 28.40 & 64.20 & 9.15 \\
\hline
\end{tabular}

FONTE: dados da pesquisa. Obs.: Valores expressos em $\mathrm{R} \$ / \mathrm{sc}$ de $60 \mathrm{kgs}$.

Observam-se na Tabela 7 as fortes reduções das oscilações da receita total com a estratégia de hedge simultâneo, indicadas pelos baixos valores do coeficiente de variação e da variância, indicando a maior eficiência do hedge simultâneo de preços e câmbio. O resultado confirma os ganhos de eficiência econômica pela utilização do hedge simultâneo, permitindo atingir-se maior mitigação de risco da receita dos produtores de soja do Centro-Oeste.

Entretanto, como é usual em estratégias de hedge usando contratos futuros, uma forte redução no risco de baixa, inferior ao valor esperado, corresponde a uma alta redução no risco de elevação, acima do valor esperado.

Com efeito, os resultados da simulação indicam que o intervalo de variação da estratégia de hedge simultâneo situa-se entre $\mathrm{R} \$ 21.04 / \mathrm{saca}$ de $60 \mathrm{kgs}$ e $\mathrm{R} \$ 48.40 / \mathrm{saca}$ de $60 \mathrm{kgs}$. Comparativamente, o intervalo da estratégia sem hedge situa-se entre $\mathrm{R} \$ 10.88 / \mathrm{saca}$ de $60 \mathrm{kgs}$ e $\mathrm{R} \$ 59.30 / \mathrm{saca}$ de $60 \mathrm{kgs}$.

Na Tabela 8 explicitam-se os decis das simulações de Monte Carlo das estratégias alternativas de hedge:

TABELA 8. DECIS DAS ESTRATÉGIAS DE HEDGE

\begin{tabular}{c|c|c|c|c}
\hline Decil & Sem Hedge & Preços & Câmbio & Simultâneo \\
\hline $10 \%$ & 21.71 & 25.90 & 21.33 & 28.36 \\
$20 \%$ & 25.28 & 28.35 & 25.10 & 29.65 \\
$30 \%$ & 27.53 & 30.00 & 27.79 & 30.71 \\
$40 \%$ & 29.61 & 31.19 & 30.13 & 31.63 \\
$50 \%$ & 31.50 & 35.00 & 32.00 & 33.80 \\
$60 \%$ & 38.20 & 34.70 & 34.22 & 30.50 \\
$70 \%$ & 36.23 & 34.62 & 36.26 & 38.30 \\
$80 \%$ & 38.47 & 35.91 & 38.54 & 34.88 \\
$90 \%$ & 46.20 & 38.22 & 40.90 & 36.17 \\
$100 \%$ & 61.06 & 47.49 & 56.86 & 45.00 \\
\hline
\end{tabular}

FONTE: dados da pesquisa. Obs.: Valores expressos em R \$/sc de 60 kgs. 
Pelos resultados da Tabela 8 confirma-se que a estratégia de hedge simultâneo domina as demais, devido ao menor valor da variância da receita total. Porém, ressalta-se que os resultados devem ser interpretados com cautela, pois a hipótese de inexistência de custos de transação poderia alterar o resultado final da estratégia de hedge simultâneo.

\section{Conclusões}

Objetivou-se comparar os resultados finais das diferentes estratégias de hedge para os produtores de soja do Centro-Oeste, particularmente de Rondonópolis (MT), com contratos futuros da BOVESPA/BM\&F, em termos de redução da variância da receita total: i. o hedge isolado dos preços; ii. o hedge isolado da taxa de câmbio; e, iii. o hedge simultâneo dos preços e da taxa de câmbio.

Como primeira etapa da pesquisa, obteve-se a relação fundamental entre as variáveis econômicas que propiciam a maior eficiência do hedge simultâneo de preços e taxa de câmbio. Observa-se que a modelagem do hedge simultâneo de preços e câmbio, deduzida no estudo, pode ser aplicável às demais commodities agropecuárias em mercados geograficamente distintos.

Em adição, calculou-se o grau de eficiência da estratégia de hedge simultâneo de preços e taxa de câmbio, expresso pela redução da variância da receita total dos produtores de soja de MT, através de testes empíricos e de simulações de Monte Carlo. Confirmou-se que a estratégia de hedge simultâneo de preços e taxa de câmbio é a mais eficiente em termos de redução de variância da receita total, resultado em linha com pesquisas similares já realizadas em outros países.

Conclui-se que os exportadores de commodities agrícolas sujeitos à exposição de risco de taxa de câmbio devem necessariamente considerar o hedge simultâneo como forma de melhorar a gestão estratégica de sua alocação produtiva total. No caso específico dos produtores de soja do Centro-Oeste observa-se uma forte redução na variância da receita total com a adoção da estratégia do hedge simultâneo. Tal fato melhora a eficiência das decisões alocativas, de produção e comercialização, ao permitir maior grau de transferência de risco.

Entretanto, para avaliar abordagens com objetivos operacionais o comportamento de mercado dos produtores de soja do Centro-Oeste sugere-se a inclusão em futuras pesquisas dos custos do hedge e a comparação da efetividade do hedge em diferentes praças de mercados futuros de soja ativos, como por exemplo, Chicago (EUA) e Tóquio (Japão). Também, as estratégias de hedge analisadas possuem características estáticas, o que pode ser refinado a partir de análise em um contexto dinâmico e simultâneo, exemplificadamente, usando o modelo GARCH-BEKK. 


\section{Referências}

BOYLE, P.; BROADIE, M.; GLASSERMAN, P. (1997). "Monte Carlo methods for security pricing". Journal of Economic Dynamics and Control, 21 (8-9).

CAMPBELL, J. Y.; LO, A. W.; MACKINLAY, A. C. (1997). The Econometrics of Financial Markets. Princeton (NJ): Princeton University Press.

CARCHANO, O.; PARDO, A. (2009). "Rolling over stock index futures contracts". Journal of Futures Markets, 29 (7); p. 684-694.

CHAMBERS, R. G.; JUST, R. (1979). "A critique of exchange rate treatment in agricultural trade”. American Journal of Agricultural Economics, 61 (2).

CHU, K. E.; MORRISON, T. K. (1984). “The 1981-82 recession and nonoil primary commodity prices”. IMF Staff Paper, 31; p. 93-140.

DALE, C. (1981). "The hedging effectiveness of currency futures markets". Journal of Futures Markets, 1 (1); p. 77-88.

FERNANDEZ, V. (2006). "Emerging derivatives markets: the case of Chile”. Emerging Markets Finance and Trade, 42(2); p. 63-92.

HULL, J. (2008). Options, Futures, and Other Derivatives. Upper Saddle River (NJ): Pearson Education, Inc..

MAIA, F. N. C. S.; AGUIAR, D. R. D. (2010). "Estratégias de hedge com os contratos futuros de soja da Chicago Board of Trade”. Gestão e Produção, 17 (3); p. 617-626.

MARTINS, A. G.; AGUIAR, D. R. D. (2004). “Efetividade do Hedge de Soja em Grão Brasileira com Contratos Futuros de Diferentes Vencimentos na Chicago Board of Trade". Revista de Economia e Agronegócio, 2(4); p. 449-472.

NAYAK, G. N.; TURVEY, C. G. (2000). “The simultaneous hedging of price risk, crop Yield Risk”. Canadian Journal of Agricultural Economics, 48(2); p. 123-140.

NOVAK, F. S.; UNTERSCHULTZ, J. R. (1966). "Simple risk measures when hedging commodities”. Journal of Futures Markets, 16(2).

ROLFO, J. (1980). “Optimal Hedging under Price and Quantity Uncertainty: The Case of a Cocoa Producer”. The Journal of Political Economy, 88(1).

THOMPSON, S. R.; BOND, G. E. (1985). "Basis and exchange rate risk in offshore futures trading”. American Journal of Agricultural Economics, 67(5).

THOMPSON, S. R.; BOND, G. E. (1987). “Offshore commodity hedging under floating exchange rates”. American Journal of Agricultural Economics, 69(1); p. 46-55.

WORKING, H. (1953). “Hedging reconsidered”. Journal of Farm Economics, 35.

Recebido em:

Aceito em: 
SOUZA, W. A. R.; MARTINES-FILHO, J. G.; MARQUES, P. V. Análise de estratégias de hedge... 